\title{
Acaricidal Daphnane Diterpenoids from Trigonostemon reidioides (KURZ) Craib roots
}

\author{
Noppamas Soonthornchareonnon, ${ }^{*}, a$ Monnun Sakayarojkul,${ }^{a}$ Masahiko Isaka, ${ }^{b}$ \\ Vanna MahakitTikun, ${ }^{c}$ Wongsatit Chuakul, ${ }^{d}$ and Prapai Wongsinkongman ${ }^{e}$ \\ ${ }^{a}$ Department of Pharmacognosy, Faculty of Pharmacy, Mahidol University; Bangkok 10400, Thailand: ${ }^{b}$ National Center \\ for Genetic Engineering and Biotechnology (BIOTEC); Pathumthani 12120, Thailand: ' Department of Parasitology, \\ Faculty of Medicine Siriraj Hospital, Mahidol University; Bangkok 10700, Thailand: 'Department of Pharmaceutical \\ Botany, Faculty of Pharmacy, Mahidol University; Bangkok 10400, Thailand: and ${ }^{e}$ Department of Medical Sciences, \\ Ministry of Public Health; Bangkok 11000, Thailand.
}

Received September 22, 2004; accepted November 8, 2004

A new daphnane diterpenoid, rediocide F (1), was isolated together with three known compounds, rediocides A (2), C (3) and E (4), from the hexane extract of Trigonostemon reidioides roots by bioassay-guided fractionation for acaricidal activity on Dermatophagoides pteronyssinus, Thai common house dust mite. The structure of rediocide $F(1)$ was established as the demethyl analog of rediocide $C(3)$ on the basis of spectral analysis. Compounds $1-4$ exhibited potent activity against $D$. pteronyssinus with respective $\mathrm{LC}_{50}$ values of $2.53,0.78,5.59$ and $0.92 \mu \mathrm{g} / \mathrm{cm}^{2}$.

Key words Trigonostemon redioides; acaricidal activity; rediocide; daphnane diterpenoid

House dust mite (HDM) is among the most important causative agent in the etiology of allergy, which is now considered to be a world wide problem. ${ }^{1)}$ Present method to control HDM is to kill the mites or reducing HDM allergen. ${ }^{2-4)}$ Several synthetic acaricides, such as benzyl benzoate, ${ }^{5)}$ phenyl salicylate and pyrethroids, have been used for controlling HDM; however, only a few studies concentrate on the search for new chemical class of acaricides from natural sources. $^{6-10)}$ In our search for novel acaricidal compounds from Thai medicinal plants, we came across the potent activity of the roots of Trigonostemon reidioides (KURZ) CRAIB (Euphorbiaceae; Thai name, Lot-tha-nong) whose extracts have been used in traditional medicine as antidote, expectorant and laxative. Previous phytochemical studies on this plant include the isolation of trigonostemone (a phenanthrenone), ${ }^{11)}$ lotthanongine (an alkaloid), ${ }^{12)}$ and rediocides A-E (daphnane diterpenoids). ${ }^{13,14)}$

The hexane extract of $T$. reidioides roots, the most acaricidal active crude extract, was chromatographed on a column of silica gel, followed by a combination of reversed phase LPLC and preparative HPLC to furnish a new rediocide ana$\log$, rediocide $\mathrm{F}(\mathbf{1})$, along with three known compounds, rediocides A (2), C (3) and E (4). Spectral data for 2, 3 and 4 were identical to literature data in all respects $\left({ }^{1} \mathrm{H}\right.$ - and ${ }^{13} \mathrm{C}$ NMR, MS, IR, and UV).

Rediocide F (1) was obtained as white powder. The IR and UV spectra of $\mathbf{1}$ were very similar to those of rediocide C (3). The molecular formula of $\mathbf{1}$ was determined by HR-MS (ESI-TOF) and ${ }^{13} \mathrm{C}-\mathrm{NMR}$ as $\mathrm{C}_{45} \mathrm{H}_{52} \mathrm{O}_{13}$, which revealed that it comprised one methyl group less than that of 3. ${ }^{1} \mathrm{H}$ - and ${ }^{13} \mathrm{C}$-NMR spectra of rediocide $\mathrm{F}(\mathbf{1})$ were similar to those of 3 except for the absence of signals for the C-19 methyl and the $\mathrm{C}-2$ methine in $\mathbf{1}$ and the additional one methylene $\left(\delta_{\mathrm{C}}\right.$ $\left.29.10 ; \delta_{\mathrm{H}} 2.02,1.38\right)$, which was assigned to $\mathrm{C}-2$ by the COSY, HMQC and HMBC correlations with H-1, H-2, H-3 and $\mathrm{H}-10$. The COSY spectrum revealed the correlation between $\mathrm{H}-1\left(\delta_{\mathrm{H}} 2.48\right)$ and $\mathrm{H}-2\left(\delta_{\mathrm{H}} 1.38\right), \mathrm{H}-3$ and $\mathrm{H}-2\left(\delta_{\mathrm{H}}\right.$ $1.38), \mathrm{H}-10$ and $\mathrm{H}-1\left(\delta_{\mathrm{H}} 2.48\right)$. The HMBC correlation no- tably from $\mathrm{H}-1\left(\delta_{\mathrm{H}} 2.02\right)$ to $\mathrm{C}-3$ and $\mathrm{C}-4, \mathrm{H}-2\left(\delta_{\mathrm{H}} 2.02\right)$ to $\mathrm{C}-$ 4 and $\mathrm{C}-10, \mathrm{H}-2\left(\delta_{\mathrm{H}} 1.38\right)$ to $\mathrm{C}-3$ and $\mathrm{C}-4, \mathrm{H}-3$ to $\mathrm{C}-1$ and $\mathrm{C}-$ 2, and that from $\mathrm{H}-10$ to $\mathrm{C}-1, \mathrm{C}-2$ and $\mathrm{C}-4$ (Fig. 1). In addition, proton and carbon chemical shifts of this moiety in $\mathbf{1}$
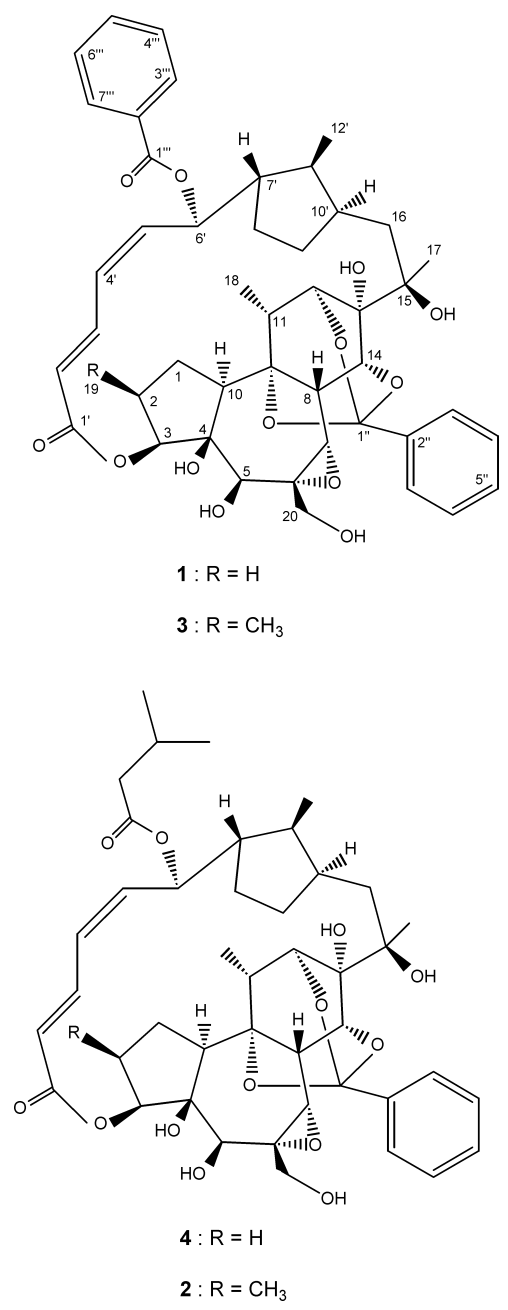

(C) 2005 Pharmaceutical Society of Japan 
Table 1. NMR Data for Rediocide $\mathrm{F}(\mathbf{1})$ in $\mathrm{CDCl}_{3}\left(500 \mathrm{MHz}\right.$ for ${ }^{1} \mathrm{H}$, and $125 \mathrm{MHz}$ for $\left.{ }^{13} \mathrm{C}\right)$

\begin{tabular}{|c|c|c|c|c|c|}
\hline Position & $\delta_{\mathrm{C}}$, mult. & $\delta_{\mathrm{H}}$, mult., $J$ in $\mathrm{Hz}$ & Position & $\delta_{\mathrm{C}}$, mult. & $\delta_{\mathrm{H}}$, mult., $J$ in $\mathrm{Hz}$ \\
\hline 1 & $29.37(t)$ & $2.48(\mathrm{~m}), 2.02(\mathrm{~m})$ & $4^{\prime}$ & $130.70(d)$ & $6.29(\mathrm{dd}, 11.4,11.4)$ \\
\hline 2 & $29.10(t)$ & $2.02(\mathrm{~m}), 1.38(\mathrm{~m})$ & $5^{\prime}$ & $136.60(\mathrm{~d})$ & $5.69(\mathrm{dd}, 10.5,10.5)$ \\
\hline 3 & $79.71(\mathrm{~d})$ & $5.09(\mathrm{~d}, 3.9)$ & $6^{\prime}$ & $73.96(d)$ & $5.51(\mathrm{dd}, 10.6,10.2)$ \\
\hline 4 & $82.14(\mathrm{~s})$ & & $7^{\prime}$ & $51.48(\mathrm{~d})$ & $2.06(\mathrm{~m})$ \\
\hline 5 & $73.21(\mathrm{~d})$ & $3.91(\mathrm{brs})$ & $8^{\prime}$ & $27.33(\mathrm{t})$ & $1.95(\mathrm{~m}), 1.29(\mathrm{~m})$ \\
\hline 6 & $60.36(\mathrm{~s})$ & & $9^{\prime}$ & $31.71(\mathrm{t})$ & $1.62(\mathrm{~m}), 1.43(\mathrm{~m})$ \\
\hline 7 & $64.58(d)$ & 3.39 (brs) & $10^{\prime}$ & $40.61(\mathrm{~d})$ & $1.70(\mathrm{~m})$ \\
\hline 8 & $35.55(d)$ & $4.73(\mathrm{~s})$ & $11^{\prime}$ & 37.55 (d) & $1.89(\mathrm{~m})$ \\
\hline 9 & $78.11(\mathrm{~s})$ & & $12^{\prime}$ & $17.01(q)$ & $0.83(\mathrm{~d}, 7.2)$ \\
\hline 10 & 48.77 (d) & $2.76(\mathrm{dd}, 13.9,5.9)$ & $1^{\prime \prime}$ & $108.83(\mathrm{~s})$ & \\
\hline 11 & $37.00(d)$ & $2.97(\mathrm{q}, 6.7)$ & $2^{\prime \prime}$ & $138.81(\mathrm{~s})$ & \\
\hline 12 & $84.53(d)$ & $3.91(\mathrm{brs})$ & $3^{\prime \prime}, 7^{\prime \prime}$ & $125.20(\mathrm{~d})$ & $7.72(\mathrm{dd}, 7.2,1.0)$ \\
\hline 13 & $71.70(\mathrm{~s})$ & & $4^{\prime \prime}, 6^{\prime \prime}$ & $128.18(\mathrm{~d})$ & $7.37(\mathrm{~m})$ \\
\hline 14 & $80.80(d)$ & $4.21(\mathrm{~s})$ & $5^{\prime \prime}$ & $129.36(\mathrm{~d})$ & $7.37(\mathrm{~m})$ \\
\hline 15 & $76.17(\mathrm{~s})$ & & $1^{\prime \prime \prime}$ & $165.83(\mathrm{~s})$ & \\
\hline 16 & $36.43(\mathrm{t})$ & $2.33(\mathrm{~d}, 15.7), 1.53(\mathrm{~m})$ & $2^{\prime \prime \prime}$ & $130.42(\mathrm{~s})$ & \\
\hline 17 & $28.35(q)$ & $1.38(\mathrm{~s})$ & $3^{\prime \prime \prime}, 7^{\prime \prime \prime}$ & $129.66(d)$ & $8.03(\mathrm{~d}, 7.9)$ \\
\hline 18 & $18.68(q)$ & $1.62(\mathrm{~d}, 6.4)$ & $4^{\prime \prime \prime}, 6^{\prime \prime \prime}$ & $128.39(\mathrm{~d})$ & $7.44(\mathrm{dd}, 7.8,7.6)$ \\
\hline 19 & - & & $5^{\prime \prime \prime}$ & $133.00(\mathrm{~d})$ & $7.56(\mathrm{t}, 7.2)$ \\
\hline 20 & $64.10(t)$ & $3.83(\mathrm{~d}, 12.6) ; 3.67(\mathrm{~d}, 12.6)$ & $4-\mathrm{OH}$ & & $3.16(\mathrm{~s})$ \\
\hline $1^{\prime}$ & $168.90(\mathrm{~s})$ & & $5-\mathrm{OH}$ & & $3.67(\mathrm{~s})$ \\
\hline $2^{\prime}$ & 124.84 (d) & $5.95(\mathrm{~d}, 15.0)$ & $13-\mathrm{OH}$ & & $3.78(\mathrm{~s})$ \\
\hline $3^{\prime}$ & 139.02 (d) & $7.81(\mathrm{dd}, 14.6,11.8)$ & $15-\mathrm{OH}$ & & $1.44(\mathrm{~s})$ \\
\hline
\end{tabular}
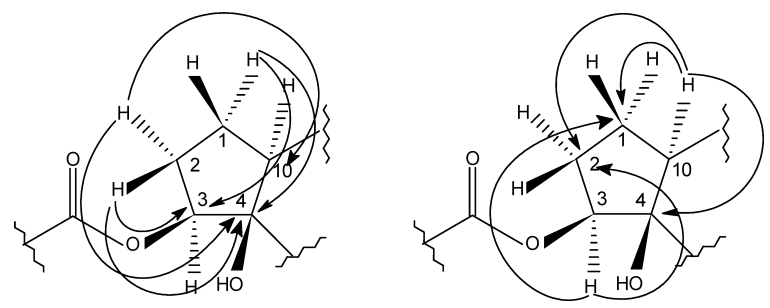

Fig. 1. Selected HMBC Correlations for Rediocide F (1)

were in comparable range to those of rediociode $\mathrm{E}(4)$, which is a 2-demethyl analog of rediocide A (2). On the basis of these spectral data, rediocide F (1) was identified as the 2demethyl analog of rediocide C. All NOESY correlation data found for $\mathbf{1}$ were similar to those of known compounds $\mathbf{2}-\mathbf{4}$, which suggested that the macrocyclic compound 1 possessed identical relative stereochemistry. Full assignments of protons and carbons for $\mathbf{1}$, established by combined analysis of COSY, HMQC, HMBC and NOESY spectra, are shown in Table 1.

Acaricidal activity on $D$. pteronyssinus of $T$. reidioides roots extracts and isolated compounds, $\mathbf{1}-\mathbf{4}$, is shown in Table 2. The $\mathrm{LC}_{50}$ values of each sample decreased in observation after 3,5 and $7 \mathrm{~d}$ of incubation time, which indicated slow response when compared to the standard acaricide, benzyl benzoate. However, at the $7 \mathrm{~d}$ observation, the four isolated compounds, 1-4, showed significant killing effect against $\mathrm{HDM}$ with respective $\mathrm{LC}_{50}$ values of $2.53,0.78,5.59$ and $0.92 \mu \mathrm{g} / \mathrm{cm}^{2}$, which are more potent than benzyl benzoate $\left(\mathrm{LC}_{50} 6.6 \mu \mathrm{g} / \mathrm{cm}^{2}\right)$.

This is the first report on the acaricidal acitivity of T. reidioides on house dust mite, and rediocides were identified as active principles in this plant. Rediocides $\mathrm{A}-\mathrm{E}$ are known to exhibit insecticidal activities against mosquito larvae (Aedes aegypti) and ectoparasitic flea (Ctenocephalides felis). ${ }^{13,14)}$ The potent acaricidal activity and structural uniqueness of
Table 2. Acaricidal Activity on Dermatophagoides pteronyssinus of T. reidioides Extracts and Rediocides 1-4

\begin{tabular}{lccc}
\hline \hline \multirow{2}{*}{ Compound } & \multicolumn{3}{c}{$\mathrm{LC}_{50}\left(\mu \mathrm{g} / \mathrm{cm}^{2}\right)$} \\
\cline { 2 - 4 } & $3 \mathrm{~d}$ & $5 \mathrm{~d}$ & $7 \mathrm{~d}$ \\
\hline Benzyl benzoate $^{a)}$ & 7.2 & 6.9 & 6.6 \\
$\mathrm{Hexane} \mathrm{extract}_{\mathrm{CH}_{2} \mathrm{Cl}_{2} \text { extract }}$ & 310 & 150 & 80 \\
MeOH extract $_{\text {Rediocide F (1) }}$ & 1140 & 210 & 110 \\
Rediocide A (2) & 9.49 & 2.19 & 0.92 \\
Rediocide C (3) & 5.40 & 6.10 & 2.53 \\
Rediocide E (4) & 16.55 & 5.04 & 0.78 \\
\end{tabular}

a) Standard acaricidal compound.

the compounds deserve further investigation.

\section{Experimental}

General Procedures Melting point (mp) were determined on Electrothermal 9100 (uncorrected). Optical rotations were recorded on a JASCO DIP-370 polarimeter. IR and UV spectra were measured on SHIMADZU 440 FT-IR and MILTONROY 3000 ARRAY spectrophotometers, respectively. NMR spectra were obtained on BRUKER AV500D and DRX400 spectrometers. The chemical shifts are given in $\delta$ with TMS (tetramethylsilane) as an internal reference. Mass spectra were obtained on a Micromass LCT mass spectrometer. Merck silica gel no. 9385 (230 - 400 mesh) was used for column chromatography. LPLC was performed on a ISCO instrument. Preparative HPLC was performed on a WATERS 600 instrument (WATERS 996 photodiode array detector).

Plant Material Roots of T. reidioides were collected in October 2001 from Yasothon Province, Thailand. A voucher specimen (Wong 2348) is deposited at Department of Pharmaceutical Botany, Faculty of Pharmacy, Mahidol University.

Extraction and Isolation The dried, ground roots $(2.8 \mathrm{~kg})$ of T. reidioides were extracted in a soxhlet extractor by hexane, $\mathrm{CH}_{2} \mathrm{Cl}_{2}$ and $\mathrm{MeOH}$, respectively. The hexane extract was found to be most active with a $\mathrm{LC}_{50}$ value of $80 \mu \mathrm{g} / \mathrm{cm}^{2}$ in the bioassay. The hexane extract $(8.0 \mathrm{~g})$ was chromatographed on a silica gel column, eluted with a gradient of hexane, $\mathrm{CH}_{2} \mathrm{Cl}_{2}$, EtOAc and $\mathrm{MeOH}$, respectively, giving 9 fractions (A-I). The active I fraction $\left(\mathrm{LC}_{50} 80 \mu \mathrm{g} / \mathrm{cm}^{2}\right)$ was re-chromatographed under the same conditions, giving 5 fractions (I1-I5). The active I2 fraction $\left(155 \mathrm{mg}, \mathrm{LC}_{50}\right.$ 
$4.02 \mu \mathrm{g} / \mathrm{cm}^{2}$ ) was subjected to LPLC (Lichroprep RP-18, $10 \times 240 \mathrm{~mm}$; flow rate, $1 \mathrm{ml} / \mathrm{min}$; detection, $\mathrm{UV}$ absorption at $254 \mathrm{~nm}$ ) with the mobile phase $\mathrm{H}_{2} \mathrm{O}$ /acetonitrile $(5: 95)$, giving a mixture of compounds $\mathbf{1}-\mathbf{4}(66 \mathrm{mg})$, which was applied to separation by preparative HPLC (Nova-Pak HRC ${ }_{18}$, $40 \times 100 \mathrm{~mm}$; flow rate, $20 \mathrm{ml} / \mathrm{min}$; detection, UV absorption at $254 \mathrm{~nm}$ ) with the mobile phase $\mathrm{H}_{2} \mathrm{O}$ /acetonitrile $(30: 70)$ yielded compounds 1 $\left(14.9 \mathrm{mg}, t_{\mathrm{R}} 25.0 \mathrm{~min}\right), \quad 2\left(17.9 \mathrm{mg}, t_{\mathrm{R}} 28.5 \mathrm{~min} ;[\alpha]_{\mathrm{D}}^{30}=-14^{\circ}, c=0.1\right.$, $\left.\mathrm{CH}_{3} \mathrm{OH}\right), 3\left(6.0 \mathrm{mg}, t_{\mathrm{R}} 30.0 \mathrm{~min} ;[\alpha]_{\mathrm{D}}^{30}=-46^{\circ}, c=0.1, \mathrm{MeOH}\right)$ and 4 $\left(10.1 \mathrm{mg}, t_{\mathrm{R}} 35.0 \mathrm{~min} ;[\alpha]_{\mathrm{D}}^{30}=-124^{\circ}, c=0.1, \mathrm{MeOH}\right)$.

Rediocide F (1): White powder; $\mathrm{mp} 199-200^{\circ} \mathrm{C} ;[\alpha]_{\mathrm{D}}^{30}:-292.0^{\circ}(c=0.1$, $\mathrm{MeOH})$; UV (MeOH): $\lambda_{\max }(\varepsilon)=236(19600), 256(21200) \mathrm{nm}$; IR $(\mathrm{KBr})$ $v_{\max }=3457,2960,1713,1602,1452,1328,1269 \mathrm{~cm}^{-1}$; HR-MS (ESI-TOF): $m / z=823.3318[\mathrm{M}+\mathrm{Na}]^{+}\left(\right.$Calcd for $\left.\mathrm{C}_{45} \mathrm{H}_{52} \mathrm{O}_{13} \mathrm{Na}, 823.3306\right) ;{ }^{1} \mathrm{H}-$ and ${ }^{13} \mathrm{C}-$ NMR: see Table 1.

Rediocide A (2): White powder; $\mathrm{mp} 193-195^{\circ} \mathrm{C}$; $[\alpha]_{\mathrm{D}}^{30}$ : $-124.0^{\circ}$ $(c=0.1, \mathrm{MeOH}) ; \mathrm{UV}(\mathrm{MeOH}): \lambda_{\max }(\varepsilon)=256(23000) \mathrm{nm}$; IR $(\mathrm{KBr})$ : $v_{\max }=3466,2960,1730,1692,1453,1288,1088,1029 \mathrm{~cm}^{-1}$; HR-MS (ESITOF): $m / z=817.3793[\mathrm{M}+\mathrm{Na}]^{+}\left(\mathrm{Calcd}\right.$ for $\left.\mathrm{C}_{45} \mathrm{H}_{52} \mathrm{O}_{13} \mathrm{Na}, 817.3775\right)$.

Rediocide C (3): White powder; $\mathrm{mp} 191-193^{\circ} \mathrm{C} ;[\alpha]_{\mathrm{D}}^{30}:-46.0^{\circ}(c=0.1$, $\mathrm{MeOH}) ; \mathrm{UV}(\mathrm{MeOH}): \lambda_{\max }(\varepsilon)=234$ (20600), $257(20400) \mathrm{nm}$; IR $(\mathrm{KBr})$ : $v_{\max }=3420,2960,1717,1603,1453,1268,1107,1029 \mathrm{~cm}^{-1}$; HR-MS (ESITOF): $m / z=837.3478[\mathrm{M}+\mathrm{Na}]^{+}\left(\mathrm{Calcd}\right.$ for $\left.\mathrm{C}_{45} \mathrm{H}_{52} \mathrm{O}_{13} \mathrm{Na}, 837.3462\right)$.

Rediocide E (4): White powder; $\mathrm{mp} 197-198^{\circ} \mathrm{C} ;[\alpha]_{\mathrm{D}}^{30}:-14.0^{\circ}(c=0.1$, $\mathrm{MeOH}) ; \mathrm{UV}(\mathrm{MeOH}): \lambda_{\max }(\varepsilon)=256(15500) \mathrm{nm}$; IR $(\mathrm{KBr}): v_{\max }=3451$, 2958, 1729, 1692, 1452, 1335, 1288, 1119, $1026 \mathrm{~cm}^{-1}$; HR-MS (ESI-TOF): $m / z=803.3642[\mathrm{M}+\mathrm{Na}]^{+}\left(\right.$Calcd for $\left.\mathrm{C}_{45} \mathrm{H}_{52} \mathrm{O}_{13} \mathrm{Na}, 803.3619\right)$.

Bioassay The procedure of acaricidal activity was modified by Mahakittikun and et al. ${ }^{15)}$ Acaricidal activity was tested on D. pteronyssinus, which have been continuously cultured at Department of Parasitology, Faculty of Medicine, Siriraj Hospital, Mahidol University. The mites were cultured in rat meal powder medium under controlled temperature $\left(25^{\circ} \mathrm{C}\right)$ and $75 \%$ relative humidity with saturated sodium chloride solution. Experiments were performed in the well of Siriraj Chamber (capacity $0.79 \mathrm{~cm}^{2} /$ well). Ten mites were placed in each well, then applied $45 \mu \mathrm{l}$ of the samples (in $20 \%$ acetone, $0.28-28 \mu \mathrm{g} / \mathrm{cm}^{2}$ for the compounds) on the mites. Covered the well with glass plate of Siriraj Chamber and motality was observed using stereobionocular microscope after 3,5 and $7 \mathrm{~d}$ incubation time. Mites that did not move when prodded with a fine brush were considered as dead, and percent deaths at each concentration were determined. Benzyl benzoate and $20 \%$ acetone in water were used as positive and negative control, respectively. All experiments were replicated three times. The $\mathrm{LC}_{50}$ values and $95 \%$ confident intervals were determined using Probit analysis method and calculated by Finney program.

Acknowledgements This work was supported by Mahidol University research grant.

\section{References}

1) Platts-Mills T. A. E., J. Allergy Clin. Immunol., 89, 1046-1059 (1992).

2) Ehnert B., Lau-Schadendorf S., Weber A., Buettner P., Schou C. Wahn U., J. Allergy Clin. Immunol., 90, 135-138 (1992).

3) Mitchell E. B., Wilkins S., McCallum D. J., Platts-Mills T. A. E., Clinical Allergy, 15, 235-240 (1985).

4) McDonald L. G., Tovey E., J. Allergy Clin. Immunol., 90, 599-608 (1992).

5) Kalpakhoglu A. F., Ferihli A. G., Misirhgil Z., Demirel Y. S., Gurbug L., Allergy, 51, 164-170 (1996).

6) Tovey E. R., McDonald L. G., J. Allergy Clin. Immunol., 100, 464466 (1997).

7) Raynaud S., Fourneau C., Laurens A., Hocquemiller R., Loiseau P., Bories C., Planta Med., 66, 173-175 (2000).

8) McDonald L. G., Tovey E. R., J. Allergy Clin. Immunol., 92, 771-776 (1993).

9) Woodfolk J. A., Hayden M. L., Miller J. D., Gail R., Chapman M. D., Platts-Mills T. A. E., J. Allergy Clin. Immunol., 94, 19-26 (1994).

10) Seon L. H., Planta Med., 70, 279-281 (2004).

11) Kokpol U., Thebpatiphat S., Boonyaratavej S., Chedchuskulcai V., J. Nat. Prod., 53, 1148-1151 (1990).

12) Kanchanapoom T., Kasai R., Chumsri P., Kraisintu K., Yamasaki K., Tetrahedron Lett., 43, 2941—2943 (2002).

13) Jayasuriya H., Zink D. L., Singh S. B., Borris R. P., Nanakorn W., Beck H. T., Balick M. J., Goetz M. A., Slayton L., Gregorn L., Zakson M. Z., Shoop W., J. Am. Chem. Soc., 122, 4998-4999 (2000).

14) Jayasuriya H., Zink D. L., Borris R. P., Nonakorn W., Beck H. T., Balick M. J., Goetz M. A., Gregom L., Shoop W. L., Singh S. B., J. Nat. Prod., 67, 228-231 (2004).

15) Mahakittikun V., Komoltri C., Nochot H., Insung A., Soonthornchareonnon N., Wongkamchai S., Vichyanond P., J. Trop. Med. Parasitol., 26, 93-97 (2003). 